\title{
Unique palliative care needs of patients with advanced chronic kidney disease - the scope of the problem and several solutions
}

\author{
Authors: Daniel Sturgill ${ }^{\mathrm{A}}$ and Alexandria Bear ${ }^{\mathrm{B}}$
}

Patients with advanced chronic kidney disease (CKD), including end-stage renal disease (ESRD), have a lifethreatening illness complicated by high morbidity and mortality and, therefore, should be suitable candidates for early intervention by palliative care specialists. However, the average patient with CKD does not have an advanced care plan, has multiple debilitating symptoms, and does not utilise hospice care at the end of life. In this review, we outline the scope of the problem of unmet palliative care needs for patients with advanced CKD and ESRD, barriers to improving palliative care for patients with renal failure, and possible future directions for palliative nephrology.

KEYWORDS: CKD, ESRD, palliative, care models

\section{Introduction}

The World Health Organisation defines palliative care as "an approach which improves the quality of life of patients and their families facing life-threatening illness through the prevention and relief of suffering by means of early identification and impeccable assessment and treatment of pain and other problems.'. Patients with advanced chronic kidney disease (CKD), including end-stage renal disease (ESRD), have a life-threatening illness that is often identified very early and follows a heterogeneous disease course that can include a multitude of problems, be they physical, psychosocial, or spiritual. These problems are particularly prevalent in the setting of comorbid illness and at the end of life. Therefore, it appears likely that patients with advanced renal disease and particularly ESRD would benefit from the incorporation of palliative care into their routine nephrology care. Patients with CKD have many needs in the areas of symptom management, advance care planning (ACP) and endof-life care. Here, we review ESRD epidemiology, key factors for prognostication, hospice care utilisation in the ESRD population, and barriers to palliative care for patients with kidney disease.

Authors: A consultant nephrologist, Medical College of Wisconsin, Milwaukee, USA; ${ }^{B}$ consultant hospice and palliative care physician, Medical College of Wisconsin, Milwaukee, USA
Lastly, we review future palliative nephrology system designs highlighted in recent literature to help meet the unmet needs of this patient population.

\section{Epidemiological considerations}

There are more than 2.2 million people in the world who receive dialysis, and the prevalence continues to increase because of improved survival on dialysis. There are more than 120,000 and 700,000 people with CKD5 in the UK and USA, respectively. ${ }^{2}$ Over the past 60 years, the dialysis population has transitioned from a relatively healthy 'single-disease' population of patients to a population with many chronic diseases and, therefore, a high burden of associated symptoms. Dialysis has transitioned from a bridge to transplant to destination therapy for many patients who will not be eligible for transplant.

Given both the presence of multiple comorbidities and an ageing dialysis population, patients have been noted to have an increasing, distressing symptom burden. The average patient on dialysis has a mean of 7.5 symptoms, with 4.5 symptoms rated as severe, based on the Edmonton Symptom Assessment Scale. ${ }^{3}$ Despite improving mortality rates over the past few decades because of improved infection control and the movement away from dialysis through catheters, only $75 \%$ of patients on dialysis are alive 1 year after dialysis initiation, with the percentage surviving after 5 years dropping to $35 \% .{ }^{4}$ Five-year mortality rates are comparable to those of patients with localised lung cancer and congestive heart failure after myocardial infarction. ${ }^{5}$ It is notable that more patients (40\%) die from arrhythmias or cardiac arrest. ${ }^{4}$ Interestingly, there is great variation in dialysis withdrawal rates between countries, with rates as high as $18 \%$ in the USA and Canada and $2-7 \%$ in European countries. ${ }^{2}$

\section{Prognostication in ESRD}

Despite the large population of patients on dialysis with welldocumented morbidity and mortality, there are few clinical tools available that clinicians can utilise to accurately prognosticate for individual patients. In 2010, Cohen et al developed a model based on data from 449 patients on haemodialysis that relied on actuarial data along with the 'surprise question' - 'Would you be surprised if your patient died in the next 6 months?' This appeared to be an accurate tool for predicting mortality, with area under the curve of 0.87 in the derivation cohort and of 0.80 in the validation 
cohort. Interestingly, the 'surprise question' corresponded with greater mortality risk (hazard ratio (HR) 2.71) compared with other objective data, such as age (HR 1.36) or dementia (HR 2.24). ${ }^{6}$ Although the Cohen et al model appeared to be accurate, in 2017 Forzley et al found that it overestimated mortality at 6 months in a Canadian dialysis cohort. ${ }^{7}$ Therefore, the value of prognostic tools might be in identifying patients at high risk of morbidity and mortality and, therefore, who might benefit from early referral to palliative care for ACP and symptom management, rather than making a definitive prognosis for an individual patient.

One of the more challenging questions the clinician faces is determining whether their older patient would benefit from dialysis initiation. Derived from a British Columbian dialysis population over the age of 65 , Wick et al developed a new mortality prediction model that does not include the surprise question. ${ }^{8}$ Factors that predict greater mortality include greater patient age, higher estimated glomerular filtration rate (eGFR, $>15 \mathrm{~mL} / \mathrm{min}$ ) at time of dialysis initiation, heart disease, and the presence of active malignancy. Of the patients in the study cohort, $17 \%$ died within 6 months of dialysis initiation. This newer prediction model for older patients is awaiting external validation studies. When considering dialysis initiation in patients in their ninth decade of life or later, it has been shown that dialysis might not provide a survival advantage compared with a conservative, non-dialytic approach. ${ }^{9}$ In a systematic review of older patients with an average age of 76.5 years, there was similar survival for patients who pursued dialysis or conservative care at 1 year. ${ }^{10}$ More prospective data are needed in the area of morbidity and mortality of older patients initiating dialysis in the inpatient setting after acute kidney injury.

\section{Hospice and hospital utilisation in the ESRD population}

Hospices are a variable enterprise according to geographic location. Within the USA, hospices represent a plan of care focused on comfort until the end of life for patients within the last 6 months of life. This care can be delivered in several locations, including the patient's home, a skilled nursing facility, or a residential (inpatient) hospice facility. This care model is in contrast to the provision of palliative care, which can be provided at any point along the course of a serious life-limiting illness. By contrast, the European model of hospice and palliative medicine, has a less clear delineation between hospice and palliative care models. The European Association for Palliative Care created a task force to further evaluate palliative care across various European countries. ${ }^{11}$ A key goal of this task force was to homogenise terminology. In 2009, the task force published definitions of palliative care and hospice in an effort to minimise variability across European countries, which were as follows. ${ }^{12}$

> Palliative care - 'the active, total care of the patient whose disease is not responsive to curative treatment.'

> Hospice - 'care is for the whole person, aiming to meet all needs - physical, emotional, social and spiritual. At home, in day care and in the hospice, they care for the person who is facing the end of life and for those who love them.'

There is no consensus definition of a hospice within Europe. Therefore, for the purposes of this review, the US hospice model is utilised, although the authors recognise that this constrains the review.
Patients with ESRD are less likely to utilise the hospice benefit than patients with other terminal illness. Only $20 \%$ of patients with ESRD utilise hospices compared with $55 \%$ of patients with cancer and $39 \%$ of patients with heart failure. ${ }^{4}$ Hospice utilisation by patients with ESRD is usually in the last several days of life, with the average patient dying 3 days after enrolling in hospice. ${ }^{13}$ One study highlighted that $18 \%$ of patients on maintenance haemodialysis noted that they would prefer to live as long as possible even if it meant suffering, and that nearly half of patients on dialysis die in hospital compared with $35 \%$ of all Medicare beneficiaries with other severe illnesses, such as dementia and chronic obstructive pulmonary disease. ${ }^{14}$ These attitudinal considerations might explain the lower hospice utilisation by the ESRD population.

Given preferences for life prolongation in combination with lower hospice utilisation, it follows that patients with ESRD also have high inpatient acute care hospital bed days per person. The average patient with ESRD will spend nearly 2 weeks hospitalised annually which increases to approximately 1 month in the last 90 days of life. During the last 3 months of life, the median number of hospital admissions is two, with $60 \%$ of patients being admitted to the intensive care unit and 33\% undergoing invasive procedures, such as intubation. Given these interventions, there is a high healthcare cost burden for patients with ESRD at the end of life, and an increase in hospice utilisation could result in dramatic cost savings. ${ }^{4}$ When considering the high burden of symptoms, high mortality, and low hospice utilisation rates, concern has been raised that the current model of providing palliative care to patients with advanced CKD and ESRD has room for improvement. One area that is beginning to come into focus as a place for improvement is the informed consent process when starting a patient on chronic dialysis.

\section{Developing a new model of palliative nephrology}

The NHS RightCare Shared Decision Making programme aims to inform and involve patients in deciding whether to pursue dialysis treatment, and the American Society of Nephrology and the American Board of Internal Medicine's Choosing Wisely campaign advocates to not start a patient on chronic dialysis without shared decision making between the clinician, patient and their family. ${ }^{15,16}$ However, there is concern that nephrologists are not providing enough informed consent when initiating dialysis. Brennan et al noted in a study of patients on maintenance dialysis, that only $30 \%$ of patients recalled their nephrologist describing the risks and benefits of dialysis, and only $1 \%$ of patients recalled discussing the option of conservative therapy. In a Boston study of patients on dialysis, no patients reported having a discussion of prognosis and, in $60 \%$ of cases, the nephrologist stated that they would not have provided a prognosis. ${ }^{17}$ In 2014, Bristowe et al sought to establish a training program for nephrologists in ACP. In the preliminary data they collected on patient experience, the investigators identified that only $50 \%$ of patients felt that their concerns were taken seriously by their nephrologist, and less than half of patients felt that they had been sufficiently involved in treatment decisions. $^{18}$

There are multiple upstream barriers that preclude practitioners from meeting the palliative care needs of patients with CKD before dialysis. In 2018, Kurella Tamura et al highlighted three main disincentives to palliative care in the CKD population: underdeveloped models of care for seriously ill patients with CKD, 
misaligned incentives between dialysis and palliative care, and uneven access to specialty palliative care in the USA. ${ }^{19}$ A survey of renal units in the UK found that there was general acceptance of conservative, non-dialytic management of ESRD, although there was variation in its scale and application between units. ${ }^{20}$ As noted earlier, prognostication in CKD is difficult; therefore, the current American hospice model, which is designed for patients in the last 6 months of life, might not yet be appropriate patients with advanced CKD, given their somewhat unpredictable life expectancies. Nevertheless, upstream palliative care interventions would likely be advantageous. However, a significant percentage (22-28\%) of hospice utilisation for allcomers in the USA is within the last 3 days of life. ${ }^{13}$ Therefore, underutilisation of hospices for patients with ESRD might be representative of the greater trend of hospice utilisation in the USA. Additionally, current advanced CKD and pre-ESRD outpatient clinic models focus on dialysis preparation and are not as focussed on ACP. ${ }^{19}$ ACP is defined by the National Hospice and Palliative Care Organisation as 'making decisions about the care you would want to receive if you became unable to speak for yourself,' and includes, but is not limited to, creation of advance directives. ${ }^{21}$ Therefore, it logically follows that patients with ESRD have a low advance directive completion rate. Current dialysis quality metrics, such as the ESRD Seamless Care Organization Quality Incentive Program have begun incorporating ACP as a quality measure, with the goal of increasing the number of patients with advance directives and surrogate decisionmakers. Despite the inclusion of ACPs as a quality metric along with measures of patient experience, quality of life and a pain assessment, the quality metrics overall contain few measures applicable to palliative care. Lastly, there is concern that a shortage of palliative care clinicians who already are primarily regionalised in larger metropolitan areas might prohibit patients from obtaining palliative care consultation. ${ }^{19}$

Improved care models of palliative nephrology delivery could remove one of the major disincentives to palliative care in patients with CKD. The most developed model of outpatient palliative nephrology comes from Australia, where patients are followed by multidisciplinary teams comprising palliative care and nephrology specialists. The Australian model has been adapted to an American palliative-nephrology clinic, with adjustments focusing on upstream palliative care, staff education, involvement of hospitalists and dialysis nurses in the referral process, and establishing evaluation tools to measure the success of the clinic. ${ }^{22}$ As discussed earlier, nephrologists have expressed a lack of confidence with having conversations about informed consent for dialysis, prognosis and ACP. A possible source for this lack of confidence might be a lack of exposure to palliative care during fellowship training. A survey of US nephrology fellows found that the trainees felt unprepared for end-of-life care and ACP for patients with ESRD, and suggested a role for improved education in palliative care during fellowship training. ${ }^{23}$

\section{Conclusions}

In conclusion, the prevalence of ESRD continues to increase and patients with ESRD have unique and unmet palliative care needs. Patients with ESRD do not often have conversations about their prognosis and all of their care options, including conservative, non-dialytic care. ${ }^{17}$ There are several barriers to providing palliative care for patients with renal disease. Improving the integration of palliative care and nephrology by increased use of outpatient combined care models and improving training in palliative care for practicing nephrologists and trainees are several possible solutions to this pressing and multifaceted problem.

\section{References}

1 WHO. WHO definition of palliative care. Geneva: WHO, 2012.

2 Qazi HA, Chen H, Zhu M. Factors influencing dialysis withdrawal: a scoping review. BMC Nephrol 2018;19:96.

3 Bruera E, Kuehn N, Miller MH, Selmser P, Macmillan K. The Edmonton Symptom Assessment System (ESAS): a simple method of the assessment of palliative care patients. J Palliat Care 1991;7:6-9.

4 United States Renal Data System. 2017. www.usrds.org/adr.aspx.

5 Gerber Y, Weston SA, Enriquez-Sarano M et al. Mortality associated with heart failure after myocardial infarction. Circ Heart Fail 2016:9:e002460.

6 Cohen LM, Ruthazer R, Moss AH, Germain MJ. Predicting six-month mortality for patients who are on maintenance hemodialysis. Clin ] Am Soc Nephrol 2010;5:72-9.

7 Forzley B, Er L, Chiu HH et al. External validation and clinical utility of a prediction model for 6-month mortality in patients undergoing hemodialysis for end-stage kidney disease. Palliat Med 2017;32:395-403.

8 Wick JP, Turin TC, Faris PD et al. A clinical risk prediction tool for 6-month mortality after dialysis initiation among older adults. Am J Kidney Dis 2017;69:568-75.

9 Verberne WR, Geers AB, Jellema WT et al. Comparative survival among older adults with advanced kidney disease managed conservatively versus with dialysis. Clin J Am Soc Nephrol 2016;11:633-40.

10 Foote C, Kotwal S, Gallagher M et al. Survival outcomes of supportive care versus dialysis therapies for elderly patients with end-stage kidney disease: a systematic review and meta-analysis. Nephrology 2016:21:241-53.

11 Connor SR, Bermedo MCS, eds. Global atlas of palliative care at the end of life. London: Worldwide Palliative Care Alliance, 2014.

12 Radbruch L, Payne S. White Paper on standards and norms for hospice and palliative care in Europe: part 1. Eur J Palliat Care 2009:16:278-89.

13 Wachterman MW, Hailpern SM, Keating NL et al. Association between hospice length of stay, health care utilization, and medicare costs at the end of life among patients who received maintenance hemodialysis. JAMA Intern Med 2018;178:792-9.

14 Davison SN, Jhangri GS, Johnson JA. Cross-sectional validity of a modified Edmonton symptom assessment system in dialysis patients: a simple assessment of symptom burden. Kidney Int 2006:69:1621-5.

15 Cassel CK, Guest JA. Choosing wisely: helping physicians and patients make smart decisions about their care. JAMA 2012;307:1801-2

16 NHS. Shared decision making programme. NHS, 2018. www. england.nhs.uk/rightcare/shared-decision-making [Accessed 15 November 2018].

17 Brennan F, Stewart C, Burgess $\mathrm{H}$ et al. Time to improve informed consent for dialysis: an international perspective. Clin J Am Soc Nephrol 2017;12:1001-9.

18 Bristowe K, Shepherd K, Bryan L et al. The development and piloting of the Renal specific Advanced Communication Training (REACT) programme to improve advance care planning for renal patients. Palliat Med. 2014;28:360-6.

19 Kurella Tamura M, O'Hare AM, Lin E et al. Palliative care disincentives in CKD: changing policy to improve CKD care. Am J Kidney Dis 2018;71:866-73. 
20 Roderick P, Rayner H, Tonkin-Crine S et al. A national study of practice patterns in UK renal units in the use of dialysis and conservative kidney management to treat people aged 75 years and over with chronic kidney failure. Southampton: NIHR Journals Library, 2015

21 NHPCO. Advance care planning. NHPCO, 2017. www.nhpco.org/ advance-care-planning [Accessed 15 November 2018].

22 Scherer JS, Wright R, Blaum CS, Wall SP. Building an outpatient kidney palliative care clinical program. JPSM 2018;55:108-16.
23 Holley JL, Carmody SS, Moss AH et al. The need for end-of-life care training in nephrology: national survey results of nephrology fellows. Am J Kidney Dis 2003:42:813-20.

Address for correspondence: Dr Daniel Sturgill, Medical College of Wisconsin, Medicine, Division of Nephrology, 8701 Watertown Plank Road, Milwaukee, Wisconsin, 53226, USA. Email: dsturgill@mcw.edu

\section{Royal College of Physicians}

\section{Physicians and global health}

The final volume of the RCP500 book series explores the influence that members and fellows of the RCP have had on global health - from the college's 16th-century beginnings to the present day - with doctors describing their experiences in fields ranging from diving medicine in Australia to researching altitude sickness in South America.

\section{Order by phone or online}

Tel +44(0)2030751358 or visit www.rcplondon.ac.uk/shop

Price: $£ 12$ (Postage and packaging not included) $10 \%$ discount for fellows and members,

$15 \%$ discount for foundation doctors and students

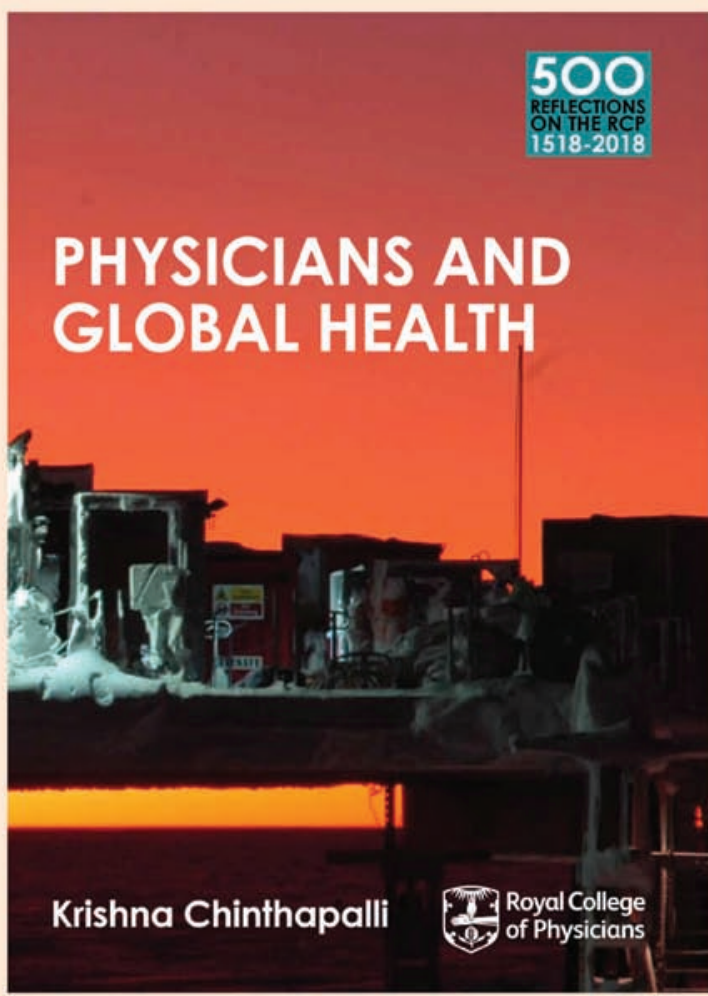

